\section{PSICOLOGÍA IBEROAMERICANA}

\section{Psicología lberoamericana}

ISSN: 1405-0943

psicología.iberoamericana@uia.mx

Universidad Iberoamericana, Ciudad de

México

México

Torres Cruz, María de Lourdes; Ruiz Badillo, Armando Motivación al logro y el locus de control en estudiantes resilientes de bachillerato del Estado de México

Psicología Iberoamericana, vol. 20, núm. 2, julio-diciembre, 2012, pp. 49-57

Universidad Iberoamericana, Ciudad de México

Distrito Federal, México

Disponible en: http://www.redalyc.org/articulo.oa?id=133928816007

- Cómo citar el artículo

- Número completo

- Más información del artículo

Página de la revista en redalyc.org

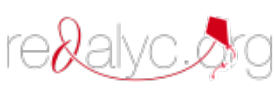

Sistema de Información Científica

Red de Revistas Científicas de América Latina, el Caribe, España y Portugal Proyecto académico sin fines de lucro, desarrollado bajo la iniciativa de acceso abierto 


\title{
Motivación al logro y el locus de control en estudiantes resilientes de bachillerato del Estado de México
}

\author{
Achievement Motivation and Locus of Control in High School \\ Students Resilient on State of Mexico
}

\author{
María de Lourdes Torres Cruz* \\ Instituto Superior de Ciencias de la Educación del Estado de México \\ Armando Ruiz Badillo** \\ Universidad Pedagógica Nacional, Unidad Ajusco
}

\section{RESUMEN}

La resiliencia es un conjunto de habilidades que desarrolla el individuo de manera positiva para poder solucionar y resolver situaciones adversas, disminuyendo los factores de riesgo e incrementando los factores protectores. Con frecuencia, los adolescentes son vulnerables porque están inmersos en diversas problemáticas psicosociales. El propósito de esta investigación fue indagar si estudiantes de educación media superior del Estado de México, identificados como resilientes (que hayan experimentado sucesos de vida estresores y muestren un buen desempeño académico), presentan mayor motivación al logro y locus de control interno que estudiantes no resilientes. Participaron en el estudio un total 464 alumnos de las escuelas preparatorias estatales en Chimalhuacán y Chalco, del Estado de México. Estas capacidades se evaluaron mediante dos escalas. Se identificaron 68 alumnos resilientes, mismos que presentan promedios más altos en la motivación al logro y el locus de control interno.

Palabras clave: resiliencia, estudiantes de bachillerato, motivación al logro, locus de control.

\section{ABSTRACT}

Resilience is a set of skills that the individual develops positively in order to troubleshoot and resolve adverse situations, reducing risk factors and increasing protective factors. Teenagers often are vulnerable by being immersed in various psychosocial problems. The purpose of this research was to investigate whether high school students than the State of Mexico, identified as resilient (who have experienced stressful life events and show good academic performance), have higher achievement motivation and internal locus of control that students do not resilient. The study involved a total of 464 students in state schools Chimalhuacán and Chalco, Mexico State. Who have evaluated these capabilities using two scales. 68 students were identified resilient themselves having higher averages in achievement motivation and internal locus of control.

Keywords: resilience, High School students, achievement motivation, locus of control.

\footnotetext{
* María de Lourdes Torres Cruz. Instituto Superior de Ciencias de la Educación del Estado de México, samantalu@yahoo.com.mx

** Armando Ruiz Badillo. Universidad Pedagógica Nacional, Unidad Ajusco, Ciudad de México (55) 5630 9700, ext. 1596 ó 1379, armandoruizb@ hotmail.com
} 


\section{INTRODUCCIÓN}

Las situaciones sociales y económicas que imperan en nuestro país impactan de forma negativa a una gran mayoría de jóvenes mexicanos. Este impacto puede ser en dos vías; en la primera, (y es el peor escenario) se convierten en "Ninis", jóvenes de entre 14 y 29 años que por diversos factores ni estudian, ni trabajan (Fernández, 2010). Conforme a lo que indica Saraví (2001) el atributo común que los ubica en una misma categoría es precisamente el permanecer al margen de algunas de las instituciones clave de socialización e integración social durante esa etapa de la vida. Tal condición de exclusión los coloca en una situación de indefinición cercana a la inexistencia social.

En la otra vía, una proporción considerable de jóvenes logra insertarse en algún espacio educativo o laboral, aunque ello no garantice, por sí solo, un mejor desarrollo social y económico. Estos escenarios dan cuenta de la vulnerabilidad a que está sometido el adolescente en el México actual.

El 27\% de la población total en México está conformado por jóvenes entre 15 y 29 años (INEGI, 2012); o sea, que aproximadamente 30,443,201 personas forman este grupo etario. El Estado de México es la entidad con mayor población juvenil, al contar con más de dos millones de ellos, de los cuales 100,706 son estudiantes en el sistema de bachillerato. Si bien es la entidad con el mayor número de estudiantes inscritos en este nivel escolar, aún así, la proporción de jóvenes que acceden a este nivel educativo es muy baja, aproximadamente un $12 \%$ del total del jóvenes mexiquenses.

Los jóvenes al estar inmersos con frecuencia en diversas problemáticas, (salud física y mental, familia, economía, violencia, adicciones, delincuencia, desempleo y baja cobertura escolar), los jóvenes presentan con frecuencia una sensación de desesperanza y apatía hacia un futuro incierto, tanto en lo educativo como en lo laboral. "Como consecuencia de ello se genera en los adolescentes ausencia del proyectos de vida, baja autoestima $y$, en ocasiones desencanto frente a las expectativas presentes y futuras" (Cardozo \& Alderete, 2009, p. 150).

Ante este escenario plagado de problemáticas sociales, donde los jóvenes son susceptibles a experimentar diversos sucesos estresores de vida que les impacten de manera significativa, es de esperarse un pronóstico negativo para muchos jóvenes estudiantes; sin embargo, hay alumnos que a pesar de experimentar sucesos estresores de vida y teniendo muchos factores en contra, pueden desarrollar una mentalidad de reto, es más, obtienen un mejor desempeño académico y cuando concursan por un lugar en alguna institución superior de alta demanda (UNAM, UAM, IPN) son aceptados en la carrera que ellos eligieron.

¿Qué hace esta diferencia?, ¿por qué en condiciones desfavorables hay personas que se sobreponen a su entorno adverso, lo superan e incluso mejoran su expectativa? Desde una perspectiva psicológica que estudia a detalle los rasgos positivos de las personas, se ha identificado a individuos que a pesar de haber estado expuestos a eventos negativos en la vida, logran sobreponerse, es decir, desarrollan resiliencia (Kotliarenco, Cáceres \& Fontecilla, 1997; Manciaux, 2003; GonzálezArratia, Valdez \& Zabala, 2008; Uriarte, 2006).

Garmezy (1993) define a la resiliencia como la capacidad para recuperarse y mantener una conducta adaptativa después del abandono o la incapacidad inicial al presentarse un evento estresante. Munist, Santos, Kotliarenco, Suárez, Infante y Groberg (1998) la definen como el resultado de un balance entre factores de riesgo (eventos negativos de vida que incrementan la probabilidad de presentar problemas físicos, psicológicos o sociales), factores protectores (influencias que mejoran o alteran las respuestas individuales ante determinados riesgos de desadaptación), y la personalidad del ser humano.

González-Arratia, Valdez Medina y González Escobar (2010) precisan que la resiliencia se ha entendido como el resultado de la combinación o interacción entre los atributos del individuo (internos) y su ambiente familiar, social y cultural (externos), que posibilitan superar el riesgo y la adversidad de forma constructiva.

En consenso con estas definiciones, para el presente trabajo se define a la resiliencia como el conjunto de habilidades que desarrolla el individuo de manera positiva, para poder solucionar y resolver situaciones adversas, disminuyendo los factores de riesgo e incrementando los factores protectores.

En la vida cotidiana hay factores de riesgo con múltiples variaciones, ante lo cual los individuos se allegan factores protectores externos (medio ambiente) y desarrollan otros internos (habilidades persona- 
les), lo que implica un proceso dinámico continuo, que busca tener un equilibrio homeostático (GonzálezArratia, et al. 2010), donde exista un contrapeso equitativo. El término resiliencia se aplicaría en específico a aquellas personas que ante una mayor magnitud o cantidad de factores de riesgo, a pesar de tener un pronóstico negativo, generan habilidades que les permiten sobreponerse y adaptarse, e incluso mejorar en su devenir cotidiano.

Una buena parte de los estudios sobre resiliencia, incluido el presente, se centran en explicar el desarrollo de habilidades o características personales de índole psicológico (Manciaux, 2003; González-Arratia, et al. 2008; Cardoso \& Alderete, 2009; Villalobos Cano, 2009).

Cardozo y Alderete (2009) indican que las principales características de personalidad que se desarrollan los jovenes identificados como resilientes, son: contar con autoconcepto positivo, autorregulación, adaptación social, competencia social, sentido del humor y empatía. Para Saavedra y Villalta (2008) los factores de índole cognoscitiva que presenta un resiliente son: la motivación de logro, el sentimiento de autosuficiencia y la confianza en que se resolverán los problemas. Adicionalmente, Becoña (2006) y Palomar y Matus (2010) coinciden en que las personas con resiliencia atribuyen el origen de su conducta a su propio esfuerzo, es decir, presentan un locus de control interno.

De la variedad de características que pueden desarrollar los jóvenes resilientes, se enfatiza la motivación al logro y el locus de control como habilidades de orden cognoscitivo que implican formas específicas de autorregulación y que tienen un fuerte impacto en el desempeño académico (Díaz, 2000; García \& ReyesLagunes, 2000; Torres-Cruz, 2009), de tal forma que si los jóvenes en condiciones de vulnerabilidad social, con antecedentes de sucesos de vida que les hayan impactado significativamente, llegan a acceder a un nivel educativo de bachillerato, por arriba del promedio nacional de escolaridad, e incluso realizan un buen desempeño escolar, están siendo resilientes y para ello han estado desarrollando habilidades propias de la motivación e internalidad. Estas características pueden contribuir en su desempeño escolar, ya que este tipo de alumnos se involucran más en sus actividades escolares, cuentan con expectativas realistas y superan situaciones difíciles (Uriarte, 2006).

\section{Motivación al logro}

La motivación es un estado interno que: "activa, dirige y mantiene la conducta, además implica impulsos o fuerzas que nos dan energía y nos dirigen a actuar de la manera en que lo hacemos" (Díaz, 2001, p. 67). En la teoría clásica tiene dos dimensiones: la motivación intrínseca, entendida como un signo de competencia y autodeterminación, y la motivación extrínseca, donde se participa en una actividad para conseguir recompensas o evitar castigos (Casas, Carranza \& Ruiz, 2011).

El estudio de la motivación intrínseca es de interés porque se desarrolla desde el interior del individuo, y tiene como objeto cumplir una meta con esmero, por ello se le denomina también motivación al logro. Para McClelland (1989) es una orientación hacia alcanzar cierto estándar de excelencia; organización, manipulación y dominio del medio físico y social. Para ello es importante la superación de obstáculos, un elevado nivel de trabajo, y establecer la competitividad por superar la propia labor, así como la rivalidad y la superación de los demás. Conforme a McClelland (1989) y Reyes-Lagunes (1998), la motivación al logro tiene tres componentes: la maestría, que es una preferencia por tareas difíciles y por intentar hacer las cosas buscando estándares de excelencia internamente prescritos; el trabajo, que representa una dimensión de esfuerzo, el deseo de trabajar duro y tener un buen desempeño, una actitud positiva ante el trabajo en sí; y como último componente, está la competencia, que se refiere a la satisfacción derivada de la competencia interpersonal y el deseo de ganar y ser mejor que los demás.

La motivación al logro se genera principalmente por intereses personales, la confianza en las capacidades propias, sin esperar nada a cambio, más que la satisfacción de haber conseguido algo por sí mismo. Se refiere a la búsqueda de una meta orientada a obtener éxito, superar los esquemas anteriores y realizar una mejor ejecución (Alonso, 2000). Es así que, en el ámbito escolar, este autor sostiene que la motivación al logro influye en la manera de pensar del alumno, las metas que pretende lograr en relación con su aprendizaje y su desempeño escolar.

Si bien el contar con una motivación al logro tiene un efecto positivo en el desempeño escolar, esta asociación es más fuerte en estudiantes considerados resilientes; así lo indica un estudio realizado por Werner 
y Smith, (1982, en Uriarte, 2006). En población adulta marginada, Palomar y Matus (2010) encontraron que los individuos con motivación al logro, específicamente en el factor de maestría, se encuentran vinculados de manera positiva con la resiliencia.

\section{Locus de control}

García y Reyes-Lagunes (2000) definen al locus de control interno como la creencia de que las conductas son contingentes a características relativamente permanentes de la persona, los cuales son responsables de sus propios éxitos y fracasos. Por el contrario, si las personas atribuyen el control de sus acciones a factores externos, se está hablando de un locus de control externo. De tal manera que el locus o lugar de control se refiere a la atribución que realizan las personas sobre el control de su conducta, si es un factor externo o un factor interno.

El locus de control es un rasgo de personalidad vinculado con la atribución que hacen los individuos de sus éxitos y fracasos. Es un constructo que se elaboró para poder explicar y describir creencias, relativamente estables, que utiliza el sujeto para fundamentar el foco de control de los sucesos diarios y de su propia conducta; de esa forma las personas diferencian el grado en el cual perciben el esfuerzo como controlado por causas internas o externas (García \& Reyes-Lagunes, 2000).

El éxito escolar se relaciona con el locus de control interno debido a que el alumno atribuye sus logros escolares a las acciones que él mismo realiza y por ende, autorregula su conducta, en la medida que quiere obtener un menor desempeño. De acuerdo con Burón (1997), una persona interna presenta mejor concepto de sí misma, una mayor autoestima, su rendimiento académico es mejor, sus aspiraciones son más altas, tiene mayor ambición, lucha más, trabaja un mayor número de horas y tiene una mayor persistencia y autoeficacia, tiende a buscar más información y a usarla para solucionar sus problemas.

Becoña (2006) realizó un análisis sobre el estilo atribucional en personas resilientes, encontrando que muestran un locus de control interno. En estudios recientes en Latinoamérica, como el de Saavedra y Villalta (2008) y el de Villalobos Cano (2009), se ha aplicado una medida de locus de control, encontrándose que las personas ubicadas como resilientes tien- den a tener puntuaciones altas en el factor de locus de control interno. También Palomar y Matus (2010) señalan que las personas resilientes perciben los eventos positivos y negativos como efecto de sus propias acciones.

En esta breve revisión teórica se señala que las personas con resiliencia desarrollan en mayor medida como características de personalidad una motivación al logro y un locus de control interno, y esto les permite obtener un mejor desempeño académico. Con ello los resilientes tienen una mayor autoestima y una mayor autoefícacia, seguridad de que pueden afrontar las situaciones exitosamente a pesar de la adversidad con la conciencia de que sus propios actos tendrán consecuencias, para modificar su entorno.

Por ello, el propósito de esta investigación fue indagar si estudiantes de educación media superior del Estado de México, identificados como resilientes (que hayan experimentado sucesos de vida estresores y muestren un buen desempeño académico), presentan mayor motivación al logro y locus de control interno que estudiantes no resilientes.

\section{MÉTODO}

\section{Participantes}

Participaron en el estudio un total 464 alumnos que cursaban primero, segundo o tercer año de bachillerato. Fueron seleccionados de forma no probabilistica accidental (Kerlinguer \& Lee, 2002). Del total de entrevistados, 219 eran alumnos de la escuela preparatoria estatal en Chimalhuacán, lo que representa un $47.2 \%$, y 245 alumnos de la preparatoria estatal en Chalco, $52.8 \%$ del total. Ambas escuelas se ubican en el Estado de México. Por sexo 172 (37.1\%) son hombres y 287 (61.9\%) mujeres, todos situados en un rango de edad de 15 a 21 años, con una mediana de 16 años.

\section{Instrumentos}

Para la presente investigación se aplicaron dos escalas: una que evalúa la motivación al logro; la otra el locus de control; además se incluyó un apartado que indaga sobre eventos estresores que haya tenido, para identificar a los participantes como resilientes o no resilientes. En otro apartado se preguntaron datos sociodemográficos. A continuación se detalla cada una de las escalas o apartados. 
Para evaluar la motivación de logro se empleó la escala diseñada para población mexicana de ReyesLagunes (1998), la cual consta de 35 reactivos redactados en forma de afirmaciones, con opciones de respuesta tipo Likert, con cinco intervalos que van de 5 "Siempre" a 1 "Nunca". Estos reactivos se agrupan en tres factores; Trabajo $(\alpha=.895)$, Maestría $(\alpha=.819)$, y Competencia $(\alpha=.862)$. La prueba, en su diseño original, ha mostrado una alta consistencia interna, con alfas de Cronbach superiores a .70.

Para evaluar el locus o foco de control se empleó la escala diseñada para población mexicana de García y Reyes-Lagunes, (2000) conformada por 23 reactivos que forman tres factores; locus de control interno $(\alpha=.86)$, locus de control externo $(\alpha=.90)$, y el factor denominado social afectivo $(\alpha=.89)$. Las afirmaciones se presentaron con opciones de respuesta tipo Likert, con cinco intervalos que van de 5 "Siempre" a 1 "Nunca". La prueba en su diseño original ha mostrado una alta consistencia interna, con alfas de Cronbach superiores a .70 .

Un punto importante en la presente investigación es poder identificar, del total de los participantes, quienes son resilientes y quienes no; para ello, un criterio es identificar a aquellas personas que refieran haber padecido un evento importante que haya afectado sus vidas, ya sea de orden físico, psicológico, social, familiar o económico. Por tanto, se realizó una versión específica para la presente investigación de un apartado denominado sucesos de vida, partiendo de dos cuestionarios; el primero diseñado para población adolescente mexicana, por Lucio, Leon, Durán, Bravo y Velasco (2001), y el segundo por Villalobos-Cano (2009), realizado en un estudio en Costa Rica. Se seleccionaron los reactivos que coincidían en ambos cuestionarios y que fueran acordes con la vida de jóvenes escolares.

La versión empleada para esta investigación cuenta con 34 indicadores sobre sucesos de vida, que abordan sucesos estresantes en las siguientes áreas: enfermedad o muerte, separación, escuela, sexualidad, actos delictivos y situación económica. Se presentó cada suceso en forma de afirmación, como "Mi familia tiene serios problemas económicos" o "Tuve un accidente que me dejó cicatrices". Las opciones de respuesta fueron; "Nunca me ha sucedido", "Sí me ha sucedido y no tuvo importancia para mí", "Sí me ha sucedido y fue malo para mí", y "Sí me ha sucedido y fue muy grave para mí".

\section{Procedimiento}

Se acudió a las escuelas y se aplicó el instrumento a los alumnos que estaban presentes en sus salones, previa aceptación para participar en el estudio, el cual consistía solamente en contestar un cuestionario impreso. También se les aclaró que los datos obtenidos serían agrupados de tal forma que se guardaría el anonimato de los participantes. Se clasificaron a los alumnos como resilientes bajo el criterio de haber tenido uno o varios sucesos de vida estresores que fueran graves para ellos (situaciones adversas) y un promedio escolar mayor de ocho (factor de protección); en caso contrario se clasificaron como no resilientes.

\section{RESULTADOS}

Entre las características sociodemográficas de todos los participantes, resalta que el $20.3 \%$ vive solamente con su mamá. En su mayoría, los padres de familia son comerciantes o tienen oficios como obreros, choferes, y albañiles. Cabe señalar que el 57.8\% (268) de las madres de los entrevistados son amas de casa. En cuanto a la escolaridad de los padres, conforme a la información proporcionada por los encuestados, $22 \%$ sólo tuvieron educación primaria, 37\% educación secundaria, $28 \%$ bachillerato y $13 \%$ tiene estudios profesionales.

Bajo los criterios previamente establecidos se identificó a 68 alumnos como resilientes, lo que representa un $14 \%$ del total de los que participaron en el estudio; 14 hombres y 54 mujeres. Las mujeres reportan más sucesos de vida estresantes que los hombres (ver tabla 1).

En un análisis detallado se encuentran rutas conflictivas; por ejemplo, hay al menos dos casos de mujeres que indicaron decepción amorosa y además un embarazo no deseado; en un caso específico un aborto y maltrato por parte de los padres. Llama la atención que siete jóvenes reportan haber sufrido abuso sexual, seis vivieron una experiencia sexual traumática o desagradable y tres tuvieron un aborto.

Otro aspecto importante se encuentra en el bloque de actos delictivos; 14 de los alumnos resilientes refieren haber padecido algún evento de asalto o secuestro, cuatro haber sido golpeados por familiares, ocho haber sido golpeados por otras personas, tres dicen tener 
Tabla 1. Sucesos de vida estresores que le han sucedido a los participantes y refieren haber sido muy graves para ellos, separados por sexo

\begin{tabular}{|c|c|c|c|c|c|}
\hline Sucesos de vida & $\begin{array}{l}\text { Núm. } \\
\text { reactivo }\end{array}$ & Categoría & Hombres & Mujeres & Total \\
\hline Fui abusada (o) sexualmente & 26 & SEX & 2 & 5 & 7 \\
\hline $\begin{array}{l}\text { Tuve alguna experiencia sexual desagradable o } \\
\text { traumática }\end{array}$ & 18 & SEX & 0 & 6 & 6 \\
\hline $\begin{array}{l}\text { Tuve un aborto provocado (si eres mujer) o mi novia } \\
\text { tuvo un aborto provocado (si eres hombre) }\end{array}$ & 4 & SEX & 0 & 3 & 3 \\
\hline $\begin{array}{l}\text { Me embaracé (si eres mujer) o embaracé a mi novia } \\
\text { (si eres hombre) }\end{array}$ & 8 & SEX & 0 & 3 & 3 \\
\hline Mi papá (mamá) se fue de la casa & 12 & SEP & 1 & 11 & 12 \\
\hline Los pleitos entre mis papás empeoran & 10 & SEP & 1 & 9 & 10 \\
\hline $\begin{array}{l}\text { Tengo algún familiar desaparecido } \\
\text { (no se sabe dónde está) }\end{array}$ & 27 & SEP & 1 & 3 & 4 \\
\hline Vivo o viví en una casa hogar & 3 & SEP & 3 & 2 & 5 \\
\hline $\begin{array}{l}\text { Me separé de mis padres para vivir con algún otro } \\
\text { familiar, novio(a), amigos(as) }\end{array}$ & 2 & SEP & 2 & 2 & 4 \\
\hline Mi familia tiene serios problemas económicos & 7 & $\mathrm{ECO}$ & 2 & 6 & 8 \\
\hline Mi papá (mamá) perdieron su empleo & 14 & ECO & 0 & 7 & 7 \\
\hline Murió mi papá (mamá), hermano(a) & 11 & MUE & 2 & 2 & 4 \\
\hline Tuve un fracaso (en la escuela) & 25 & ESC & 4 & 6 & 10 \\
\hline Tuve un accidente que me dejó cicatrices & 20 & ENF & 2 & 7 & 9 \\
\hline $\begin{array}{l}\text { Algún familiar o yo tenemos problemas en relación con } \\
\text { el alcohol o drogas }\end{array}$ & 16 & ENF & 0 & 7 & 7 \\
\hline $\begin{array}{l}\text { Algún familiar o yo padecemos alguna discapacidad } \\
\text { (motriz, mental) }\end{array}$ & 6 & ENF & 3 & 2 & 5 \\
\hline $\begin{array}{l}\text { Yo padezco alguna enfermedad física o mental grave o } \\
\text { importante }\end{array}$ & 15 & ENF & 2 & 2 & 4 \\
\hline $\begin{array}{l}\text { Mi padre, madre o hermanos, padecen alguna } \\
\text { enfermedad física o mental, grave o importante }\end{array}$ & 31 & ENF & 0 & 2 & 2 \\
\hline $\begin{array}{l}\text { Algún familiar o yo vivimos un asalto violento o } \\
\text { secuestro }\end{array}$ & 33 & ADE & 4 & 10 & 14 \\
\hline Fui golpeado(a) por algún miembro de mi familia & 19 & ADE & 1 & 3 & 4 \\
\hline Tengo uno de mis papás o hermanos(as) en la cárcel & 21 & ADE & 1 & 2 & 3 \\
\hline
\end{tabular}

SEX Sexualidad, SEP Separación, ECO Situación económica, MUE Muerte, ESC Escuela, ENF Enfermedad, ADE Actos delictivos 
familiares en la cárcel y siete alumnas refieren problemas de adicciones en casa. Lo que da cuenta que muchos de estos alumnos resilientes viven en un entorno social y familiar peligroso y hostil.

\section{Motivación al logro}

En la evaluación sobre motivación al logro que se llevó a cabo entre los participantes, al realizar comparaciones sobre los puntajes obtenidos por el grupo de estudiantes resilientes versus el grupo de estudiantes no resilientes, se encontraron diferencias estadísticamente significativas en los factores de trabajo, donde las puntuaciones en promedio son más altas en el grupo de alumnos resilientes (ver tabla 2).

Esto indica que las personas resilientes tienen una motivación al logro orientada hacia la realización de tareas, ven al trabajo con gusto y como un reto. A su vez, estos trabajos o labores están sometidos a una autoevaluación para realizar cada vez mejores ejecuciones, cuidando su perfeccionamiento. Es importante recordar que los alumnos identificados como resilientes en este trabajo tienen calificaciones promedio superiores a ocho, por lo que se puede asumir que a pesar de haber sufrido algún evento estresante importante están teniendo al menos un buen desempeño escolar.

\section{Locus de control}

Al realizar la medida de locus de control y comparar los puntajes de los factores respectivos se encontró que el grupo de alumnos con resiliencia presenta puntajes promedio más altos que los alumnos no resilientes en el factor de locus de control interno (ver tabla 3 ).

Esto muestra que los alumnos resilientes, predominantemente, atribuyen como origen de sus conductas a su propia persona, por lo que existe en ello una fuerza interna que los autoregula. Por lo tanto, muestran iniciativa, interés personal para la realización y participación de actividades, en este caso principalmente en la escuela, motivo por el cual su desempeño en este campo puede verse incrementado debido a que ellos auto dirigen sus actividades.

\section{CONCLUSIONES}

El presente estudio se realizó en una muestra de estudiantes provenientes de poblaciones que mayoritariamente tienen un nivel socioeconómico bajo, en los municipios de Chimalhuacán y Chalco, del Estado de México. El hecho de ser estudiantes a nivel bachillerato representa una ventaja en sí, porque la escuela misma puede significar un factor de protección, aún así, contar con niveles de escolaridad más altos no im-

Tabla 2. Motivación al logro por alumnos resilientes y no resilientes

\begin{tabular}{|l|c|c|c|c|c|c|}
\hline \multirow{2}{*}{ Factor } & \multicolumn{2}{|c|}{ Resiliente } & \multicolumn{2}{c|}{ No Resiliente } & Valor de t & Prob. \\
\cline { 2 - 7 } & Media & Desv. Estándar & Media & Desv. Estándar & & \\
\hline Trabajo & 3.8937 & .48922 & 3.7216 & .59546 & 2.255 & .025 \\
\hline Maestría & 4.5408 & .34496 & 4.3650 & .52052 & 2.685 & .008 \\
\hline Competencia & 2.9583 & .80878 & 2.9792 & .79359 & -.199 & .842 \\
\hline
\end{tabular}

Tabla 3. Locus de control por alumnos resilientes y no resilientes

\begin{tabular}{|l|c|c|c|c|c|c|}
\hline \multirow{2}{*}{ Factor } & \multicolumn{2}{|c|}{ Resiliente } & \multicolumn{2}{c|}{ No Resiliente } & Valor de t & Prob. \\
\cline { 2 - 7 } & Media & Desv. Estándar & Media & Desv. Estándar & & \\
\hline Interno & 4.6601 & .40400 & 4.5008 & .51113 & 2.441 & .015 \\
\hline Externo & 2.0357 & .75245 & 2.0444 & .76317 & -.087 & .931 \\
\hline Afectivo & 2.3088 & .91390 & 2.3872 & .89327 & -.666 & .506 \\
\hline
\end{tabular}


plica garantía alguna de un mejor desarrollo de vida y/o ascenso económico. Como se pudo observar, los participantes en este estudio presentan diferentes niveles de vulnerabilidad y muchos de ellos han estado sometidos a diversos sucesos de vida estresores. Cardozo y Alderete (2009) hacen referencia a diversos estudios en donde los principales factores de riesgo para el desarrollo de los jóvenes son: divorcio o pérdida de trabajo de los padres, muerte de algún familiar cercano, abuso sexual, violencia psicológica o física, y bajo nivel socioeconómico. En esta investigación, los sucesos estresores de vida y por ende, factores de riesgo que reportaron los alumnos que fueron identificados como resilientes son similares a los que indican estos autores, además, para ser clasificados como tales, la segunda condición fue que tuvieran un promedio escolar mayor a ocho, lo que indicaría que cumplen con lo establecido por la definición de resiliencia, ya que existieron participantes que reportaron eventos estresores de vida pero bajos promedios escolares, lo que mostraría sólo su vulnerabilidad y no estar desarrollando alguna habilidad resiliente.

En la evaluación de la motivación al logro los resilientes tienen promedio más alto que los no resilientes en los factores de trabajo y maestría, lo que indica que los alumnos resilientes tienen una orientación hacia alcanzar cierto estándar de excelencia; organización, manipulación y dominio del medio físico y social, les interesa superar obstáculos, tienen un elevado nivel de trabajo, y buscan realizar sus ejecuciones cada vez mejor, atributos que conforme al autor de este concepto, McClelland (1989) presentan las personas con trabajo y maestría. Esta diferencia concuerda con la evaluación obtenida por Palomar y Matus (2010), que concluyeron que las personas con resiliencia contaban con una mayor motivación al logro en el factor de maestría.

Habría que recordar lo que indica Alonso (2000) sobre la motivación al logro en estudiantes: se caracterizan por realizar preguntas en clase para aclarar sus dudas, comentan sus errores, repasan sus tareas para ver posibles equivocaciones, tienen como meta principal el aprender, independientemente de los elogios que puedan recibir.

En la medida de locus de control que está compuesta por locus de control interno externo y efectivo, se encontró que los resilientes tienen un locus de con- trol interno más alto que los no resilientes; en los otros factores no hay diferencias estadísticamente significativas, por lo que lo se puede identificar que los resilientes tienen una regulación interna y el foco de su conducta lo sitúan a partir de su propio esfuerzo. Lomelí (1999) afirma que la forma atribucional de los estudiantes se relaciona con el desempeño académico, por ello el éxito escolar tiene que ver con el locus de control interno, principalmente en la medida de que el alumno percibe las consecuencias de sus acciones como propias de él.

Este hallazgo concuerda con Becoña (2006), que afirma que el locus de control es un factor que se promueve en la resiliencia y protege de los estresores de la vida, así también con estudios realizados en Latinoamérica, como el de Saavedra y Villalta (2008) y el de Villalobos Cano (2009), y en específico en México, los de González Arratia et. al (2008), que indican que los adolescentes con resiliencia tienen un locus de control interno.

En la educación se vuelve central la posibilidad de identificar y fomentar la resiliencia de los estudiantes, para que puedan enfrentar su crecimiento e inserción social de modo favorable (Kotliarenco, Cáceres \& Fontecilla, 1997).

Identificar a alumnos en riesgo y generar en ellos capacidades de resiliencia es una prioridad que se debe tomar en cuenta en las actividades cotidianas de toda escuela, por ello es relevante incorporar en los programas educativos, actividades que ayuden a promover fortalezas internas como: habilidades para la vida, límites claros y firmes en la acción educativa, motivación al logro, creatividad e iniciativa entre otras. Además de realizar intervenciones específicas en las escuelas para identificar, por medio de encuestas similares a la empleada en el presente estudio, a alumnos resilientes, y posteriormente diseñar la intervención educativa tomando en cuenta el contexto escolar y comunitario para promover las conductas resilientes.

Otra recomendación es que la resiliencia puede ser promovida en el contexto educativo a través de educadores, orientadores, tutores, profesores, dando relevancia a proyectos enfocados a la disposición de disminuir los factores de riesgo y haciendo énfasis en la promoción de los factores protectores; también por medio de la familia, al lograr su inserción en el medio escolar y crear nexos directos entre profesores y padres de familia (Barroso \& Mendez, 2008). • 


\section{REFERENCIAS}

Alonso, I. (2000). Motivación y aprendizaje en el aula. Madrid, España: Santillana.

Barroso, R. \& Mendez, M. (2008). La resiliencia en niños para su desarrollo en la sociedad. Revistas en Ciencias de la Educación, 1(31), 269-292.

Becoña, E. (2006). Resiliencia: definición, características y utilidad del concepto. Revista de psicopatología y psicología clínica, 11(03), 125-146.

Burón, J. (1997). Motivación y aprendizaje. ( $3^{\circ}$ Edición). Bilbao, España: Ediciones Mensajero.

Cardozo, G. \& Alderete, A. (2009). Adolescentes en riesgo psicosocial y resilientes. Psicología desde el Caribe, 23, 148-182.

Carretero Bermejo, R. (2010). Resiliencia. Una visión positiva para la prevención e intervención desde los servicios sociales. Revista Crítica de Ciencias Sociales y Jurídicas, 27(No.3).

Casas Santin, V., Carranza Peña, G. \& Ruiz Badillo, A. (2011). Guía para la planeación didáctica en la Universidad. México: UPN Polvo de gis.

Castro, M. E. \& Llanes, J. (2006). Tutoría en resiliencia. LibertAddictus, 101-104.

Cayssials, A., Dana, A. \& Pérez, M. A. (2006). Estilos del sentido del humor. Un estudio transcultural en población adulta según el género. XIII Jornada de investigación en psicología del Mercosur, 32-33.

Díaz Barriga, F. (2000). La motivación escolar y sus efectos en el aprendizaje en estrategias docentes para un aprendizaje significativo. Una interpretación constructivista. México: Mc.Graw Hill.

Fernández, L.F. (2010) Entre ninis y memes.... CULCyT Cultura Científica y Tecnológica. Universidad Autónoma de Ciudad Juárez. (Núms. 38-39), 47-48.

García Campos T. \& Reyes Lagunes I. (2000) Estructura del locus de control en México. La psicología social en México. $6,158-164$.

Garmezy, N. (Feb de 1993). Children in poverty: Resilience Despite Risk. Psychiatry, 56, 127-136.

González-Arratia, N., Valdez Medina, J. \& Zabala Borja, Y. (2008). Resiliencia en adolescentes mexicanos. Enseñanza e Investigación en Psicología, 13(1), 41-52.

González-Arratia, N., Valdez Medina, J. L. \& González Escobar, S. (2010). Resiliencia y optimismo en adolescentes. La psicología social en México, XIII, 815-820.

Guillén, R. (2005). Resiliencia en la adolescencia. Revista Social de Pedagogía, 44(1), 41-43.

INEGI. Estadística básica, demografía y población. Consultado en http://www.inegi.org.mx.
Kerlinger, F. \& Lee, H. (2002) Investigación del comportamiento. Método de investigación en ciencias Sociales. México: Mc Graw Hill.

Kotliarenco, M., Cáceres, I. \& Fontecilla, M. (Julio de 1997). Estado de arte en resiliencia. Organización Panamericana de la Salud, 1-49.

Lucio, E., León, I., Durán, C., Bravo, E. \& Velasco, E. (2001). Los sucesos de vida en dos grupos de adolescentes de diferente nivel socioeconómico. Salud Mental, 24(5), 17-24.

Manciaux, M. (2003). La resiliencia: resistir y rehacerse. Gedisa.

McClelland, D. (1989). La motivación de logro, estudio de la motivación humana. Madrid, España: Nancea.

Munist, M., Santos, H., Kotliarenco, M., Suárez, E., Infante, F. \& Groberg, E. (1998). Manual de identificación y promoción de la resiliencia en niños y adolescentes. Organización Panamericana de la Salud.

Palomar, J. \& Matus, G. (2010). Contribución de los atributos personales sobre la resiliencia: El caso de la pobreza. Psicología Social en México, XII, 529-536.

Prada, E. (2010). Psicología positiva. Recuperado de http://www. psicologiapositiva.com/psicologiapos.pdf, 1-30.

Reyes-Lagunes, I. (1998) La orientación al logro, la evitación al éxito y el temor al fracaso en México. Simposio: Cultura y psicometría. Congreso Mexicano de Psicología Social. III Reunión Regional Latinoamericana de la Asociación Internacional de Psicología Transcultural. Toluca, Estado de México. 21 al 23 de octubre de 1998.

Saavedra, E. \& Villalta, M. (2008). Medición de las características resilientes, un estudio comparativo en personas entre 15 y 65 años. LIBERABIT, 14, 31-40.

Saraví, G. (2001) Entre la evasión y la exclusión social: jóvenes que no estudian ni trabajan. Aportes Nueva Sociedad. Recuperado de: http://132.248.9.1:8991/hevila/Nuevasociedad/ 2004/no190/7.pdf

Torres-Cruz, M. L. (2009). Motivación al logro y estructura de valores en estudiantes de un centro de bachillerato tecnológico del Estado de México. México: Tesis para obtener el grado de Maestra en Psicología Social. unam, Facultad de Psicología.

Uriarte, J. (2006). Construir la resiliencia en la escuela. Psicodidáctica, 11(No.1), 7-23.

Villalobos-Cano, A. (2009). Desarrollo de una medida de resiliencia para establecer el perfil psicosocial de los adolescentes resilientes. Tesis para obtención de grado de Especialista en Psicología Clínica. Universidad de Costa Rica.

Fecha de recepción: septiembre 2012 Fecha de aceptación: octubre 2012 